\title{
ADVANCES IN MODELING AND SIMULATION
}

$\mathrm{I}$ n 1999, Information \& Security: An International Journal published volume 3 that was focused on Modeling and Simulation (M\&S) techniques and their applications to security related issues including defense, internal security and international cooperation. Since that time, the use of modeling and simulation $(M \& S)$ is becoming even more pervasive throughout the NATO defense environment. The value of simulations to defense establishments has been repeatedly proven by providing readily available, operationally valid environments to train jointly, develop doctrine and tactics, formulate operational plans; assess war fighting situations; and support technology assessment, system upgrade, prototype and full-scale development, and force structuring.

To address the advances made in the field, Information \& Security has decided to publish a second issue devoted to defense modeling and simulation. We have received many papers covering the broad spectrum of topics in defense modeling and simulation.

In particular, based on the accepted papers, we have decided to organize the special journal issue on advances in modeling and simulation in two numbers. Volume 12, number 1 focuses on the following groups of topics:

- Review of Defense Modeling and Simulation

- Computer Generated Forces, and

- Agent-based Modeling and Simulation.

Number 2 deals with:

- Simulation Reuse and Interoperability

- M\&S Applications, and

- M\&S in Information Assurance.

\section{Review of Defense Modeling and Simulation}

The objective of the first group of papers is to provide a review of the achievements in defense modeling and simulation. While the first paper takes a global perspective, the second discusses the R\&D work performed in a single country - Croatia. 
Modeling and simulation are essential tools in defense planning, development and acquisition of systems, training and exercises, and operational planning. In his article, "Modeling and Simulation in Defense," Klaus Niemeyer provides a contribution to a theoretical approach to the technology, with discussion of definitions and characteristics, such as purpose of a model, reduction of complexity, and representation of real entities or systems. Klaus Niemeyer's paper discusses also specific issues in modeling of the defense system, such as the military hierarchical structure, functional areas, operational phases, planning situations, and the decision cycle.

The second paper in this group, "Combat and Security Related Modeling and Simulation in Croatia," describes the research and development activities in Croatia of state-of-the-art combat and security-related educational and training simulators. Krešimir Ćosić, Miroslav Slamić, and Dražen Penzar present individual and crew training simulators for antitank guided missiles, low altitude air defense missiles, jet fighter aircraft and hardware-in-the-loop simulators for real time testing of newly developed digital signal processing guidance and control system. Design and development work of high-resolution tactical simulations and operational aggregated combat simulations is also described. Finally, Ćosić and coworkers present a theoretical approach applied to the modeling and simulation of national power and national security.

\section{Computer Generated Forces}

Human performance in combat is a key element to success. From the humans' ability to do physical work to their ability to make sound decisions, success in combat is largely a function of the human element. Therefore, the value of computer models of combat is greatly affected by their ability to accurately represent the range and variability of expected human behavior. Computer models of complex human behavior have been around for over two decades, but their use in computer models of combat continues to be developed. The distributed simulation environments that are being used for training and analysis drive much of the new interest. Today, simulation exercises involve a few dozen real people with the remaining hundreds or even thousands of other battlefield entities being computer simulations. These computer generated forces are becoming a foundation of current and future military training and analysis.

Many researchers have worked to add realism to human representations through a variety of computer modeling techniques. And this is exactly the place where the papers in this group contribute. 
Should computer generated forces systems include automated learning capabilities? This is the question posed by Mikel D. Petty in his paper "Benefits and Consequences of Automated Learning in Computer Generated Forces Systems." The idea that CGF systems can and should include automated learning capabilities has been widely asserted and accepted. The CGF research literature contains many statements by CGF experts that the ability to learn will be generally valuable, even necessary, in future CGF systems. A variety of significant benefits for CGF systems and military simulation in general are claimed to follow from automated learning. However, according to Mikel D. Petty, it seems to be not so obvious that learning by CGF systems would necessarily be beneficial for many uses of CGF systems. The author takes a respectfully skeptical position regarding CGF learning and provides arguments that CGF learning could compromise and confound the utility of CGF systems for the most common CGF applications. For each of the three broad classes of CGF applications there are categories of learning-modified behavior for CGF systems that apparently could reduce or negate the utility of the CGF system for the application. The specific applications where learning by CGF systems might be useful are a subset of CGF applications, concludes Mikel D. Petty.

In a military simulation with automated commanders, models of command behavior and decision-making that follow doctrine precisely and exhibit no variations are unrealistic due to the uncertainties inherent in military operations and the variations in human personalities. Automated commanders that exhibit doctrinal behavior are essential for training, but are not sufficient for the full range of purposes the simulation may be applied to. Simulation users would like to have an automated commander that realistically models the effects of the fog of war and the difficulty of making doctrinal decisions under stressful conditions. Such realism in simulation could better prepare trainees for expected encounters on the battlefield.

To achieve this end, the realistic modeling of human behavior is considered as a priority research area for the M\&S community. The second paper in this group, "An Experimental Application of a Trait-Based Personality Model to the Simulation of Military Decision-Making," makes a contribution to this extremely difficult and challenging area.

How to represent realistic human behavior? Based on the suggestion of some psychologists, Rick McKenzie, Mikel Petty, and Jean Catanzaro use personality traits to characterize behavior. Personality significantly influences human behavior. In the context of military decision-making, different military commanders may behave differently in the same situation, depending on their personalities. Moreover, personality may cause the same commander to react differently to similar situations encountered at different times. 
To investigate the inclusion of personality in models of military command decisionmaking, Rick McKenzie, Mikel Petty, and Jean Catanzaro have implemented and tested a simulation wherein a simulated commander must make critical decisions under multiple pressures. The commander's behavior model allows the specification of personality using a set of eight personality traits. In general, personality traits determine the predisposition of people to exhibit a particular behavior under varying situational conditions. In their research, the authors combine the commander's personality traits and the situational conditions to produce effects such as reaction time delay and decision accuracy and effectiveness modifications. McKenzie, Petty, and Catanzaro showed that modeling personality and its effects on the decisionmaking of a commander could improve simulation realism. Their model was implemented and used in an experiment intended to test its utility in producing more realistic human decision-making in a way that could be validated by personality and performance measurements of real human commanders. Looking farther ahead, a personality model may also be applied to the task of predicting how a particular military commander might react in a situation and how to improve that commander's performance.

\section{Agent-based Modeling and Simulation}

Computer simulation is a valuable tool for complex decision-making, especially in military and civilian operations in the land, air or sea. As we have already elaborated, simulation has been used in the military domain for the evaluation of acquisitions, missions and force development options. Modeling and simulation for this purpose is becoming increasingly complex as multi-role, multi-platform and multi-system aspects are taken into consideration. The complexity of this task is further increased by the difficulty in modeling human decision-making using conventional software approaches. Current implementations of computer generated forces have proven to be very useful, but do not model human reasoning and cannot easily model team behavior. Applications of intelligent agents in military simulations have proved highly effective. This is due to the capability of agents to represent individual reasoning and from the architectural advantages of that representation to the user due to the ease of setting up and modifying operational reasoning or tactics for various studies. In addition, intelligent agents extend the modeling of reasoning to explicitly model the communications and coordination of activities required for team behavior.

The aim of the next group of papers in this special issue on advances in modeling and simulation is to point out the importance of agent-based modeling and simulation, as a scientific concept and technological possibility, to enhance the potential of simulation in both civilian and defense applications. The interested reader may refer 
to a previous issue of Information\& Security devoted especially to this important technology for modeling and simulation.

The emphasis on timely, accurate information in modern warfare, and the availability of modern communications, has led to the development of increasingly complex command and control systems. It is important to understand the behavior of these systems under a variety of circumstances. However, as they are difficult to analyze manually, advanced modeling and simulation tools for command and control systems development are required. As we have already elaborated, the challenge in these systems is to model the reasoning associated with different roles in the hierarchy. Intelligent agents can represent the reasoning and command capabilities associated with their assigned roles in the hierarchy, allowing different command and control strategies to be quickly evaluated under varying circumstances.

These intelligent agent simulation models were in the focus of the paper by James Moffat and Susan Witty published in Volume 8 of the journal. Such agent models consist of a number of entities which interact locally in order to produce global emergent behavior. In complex systems, elaborate and unpredictable properties arise from the interaction of the constituents. Examples of such emergent properties include how the system organizes itself, how it finds a balance between order and disorder, and how agents, both individually and collectively, evolve new behaviors in response to change. Some of the emergent behaviors can be surprising, and the work of James Moffat and Susan Witty published in Volume 8 of the journal described a theoretical approach to the development of mathematical "meta-models," which aim to capture the emergent behavior of intelligent agent-based constructive simulation models of military conflict. These intelligent agents capture the process of C4ISR (Command, Control, Communications, Computers, Intelligence Surveillance and Reconnaissance) in such agent-based simulation models.

The principal variables in an intelligent agent simulation models can often be separated out from the rest of the model to develop a meta-model that is aimed at decreasing the run-time of the original model while still retaining the characteristics and arriving at the same final solution as the original model. The meta-model of Moffat and Witty is a mathematical abstraction of such a simulation, composed of two parts. For the first part, the fractal dimension of a force is introduced as a parameter measuring the emergent ability of such forces to cluster locally, corresponding to local decision-making by individual agents. For the second part the authors consider the mathematics of Bayesian Decision-Making as a meta-model for top down decision processes in such simulation models.

These meta-models fall within the area of what is loosely referred to as complexity theory, and exploit the mathematical approaches which are being developed to gain 
understanding of natural non-linear systems. Such an approach is most likely to be relevant to future command and control structures such as Network Centric Warfare.

In the current paper, "Experimental Validation of Metamodels for Intelligent Agents in Conflict," the authors present both historical evidence and evidence from experiments using cellular automata models that support hypotheses derived from their theory.

As a means of gaining understanding, the authors have carried out a number of experiments using simple cellular automata based models that are relevant to conflict. Such models have been developed in response to the theory that human conflict is a complex, non-linear system, which in dynamical system terms, occurs far from equilibrium.

The 'new sciences' of complexity and chaos provide a way of looking at such interacting agents in conflict. In their paper, James Moffat and Susan Witty first show that historical data indicate the existence of a fractal attractor for at least some types of conflict. Then, the authors show that experimental data from runs of such simple cellular automata models supports the hypotheses, which can be derived from their theoretical meta-models of the process.

The second paper in this group, "Soft Computing Agents for Dynamic Routing," reviews and evaluates the state-of-the-art in Distributed Information Systems. The author Georgi Kirov outlines some disadvantages of distributed software. He concludes that the field of distributed network systems is in a critical need of intuitive and innovative approaches to address the growing complexity in all of its different aspects: communication, routing, performance, stability, and connectivity. In an attempt to resolve the above-mentioned problems the work of Georgi Kirov proposes an approach that combines the Bee-gent agent technology and the fuzzy-logic representation. The author presents an example of soft-computing agents for dynamic routing that uses distributed database applications as illustration of the concept.

\section{Simulation Reuse and Interoperability}

Interoperability is an operationally driven requirement in several application domains of combat simulation systems and it is stated in milestone documents such as the U.S. DoD Modeling and Simulation Master Plan and the NATO Modeling and Simulation Master Plan.

While the use of modeling and simulation for military purposes is expanding, recent work by the NATO Steering Group for Modeling and Simulation has demonstrated that most applications in the NATO nations have been developed by individual organizations to meet the explicit needs of a particular user community; are not 
integral to operational systems; take too long to build and cost too much; can not be used in joint applications and are not fully validated. The consequence was the proposal to develop and apply standards and interoperability procedures as provided by the High Level Architecture (HLA).

The High Level Architecture is architecture for constructing distributed simulations. It facilitates interoperability among different simulations and simulation types and promotes reuse of simulation software modules. HLA addresses a number of the limitations imposed by the data protocol approach associated with the earlier Distributed Interactive Simulation (DIS) standard. HLA has been mandated by the U.S. Department of Defense, has been published as a standard by the Institute of Electrical and Electronics Engineers (IEEE) and the Object Management Group $(\mathrm{OMG})$, and is being adopted by creators of simulation software worldwide. HLA can support virtual, constructive, and live simulations from the training, engineering, and analysis applications domains. While in many respects HLA achieves these goals, it unfortunately also adds a significant amount of overhead and complexity to the development process, resulting in the need for specialist HLA skills; a lot of extra work and code is needed to build the necessary software infrastructure needed for HLA compliance.

Shawn Parr's paper, "A Visual Tool to Simplify the Building of Distributed Simulations Using HLA" outlines the problems currently faced by simulation developers wanting to use HLA, and introduces Calytrix SIMplicity product to address them. Shawn Parr presents SIMplicity, which delivers an Integrated Development Environment (IDE) HLA development, based on the OMG's Model Driven Architecture, to simplify the process of developing distributed simulations. SIMplicity enables software developers and scientists to rapidly create large-scale, high fidelity component-based simulations from new and pre-existing components in a visual environment. The product makes it feasible for developers to create HLA simulations without specialist HLA or middleware knowledge.

Due to historic constraints, the two military IT families of Modeling and Simulation $(\mathrm{M} \& S)$ and Command, Control, Communications, Computers, Intelligence, Surveillance, Reconnaissance (C4ISR) were more or less developed separately. For integrated support for the needs of modern armies, however, a combination of both sides' functionality is needed. The motivation for improving the interoperability between simulations and C4ISR systems include: simulation based acquisition; development of doctrine and tactics techniques, and procedures; computer assisted exercises; embedded training; course of action development and analysis; mission planning and rehearsal; monitoring execution; and command and control. Furthermore, future military operations require interagency interoperability to enable cooperation of various national/international partners and will be conducted in 
joint/combined environments, often in tight collaboration with non-military and nongovernmental organizations. As it is very unlikely that these organizations and international partners will use U.S. DoD standards to implement their information systems, there need to be well-understood ways to insure information exchange with such systems.

Therefore, there is a strong need to solve the interoperability issue between information systems used for Command, Control, Computing, Communications, Intelligence, Surveillance, and Intelligence (C4ISR) and combat simulation systems. A step in this direction is taken in the contribution by Andreas Tolk.

The solution to couple the simulation system delivering the needed functionality with the Command, Control, Computing, Communications, Intelligence, Surveillance, and Reconnaissance (C4ISR) system providing the necessary data is to build appropriate interfaces. Although in long term a more integrated approach will be necessary, in short and mid term, gateways and interfaces are likely to remain the standard. However, as Andreas Tolk claims, in order to succeed with the respective efforts at least on the data level of interoperability, a common solution is necessary. Subsequently, in achieving interoperability issues like a common architecture, a common set of algorithms, and a common view of the world in the form of ontology, including dynamic aspects, can be addressed as well. First of all, however, in order to make a meaningful integration possible, the common data issue has to be dealt with. The methods used to achieve this are not only applicable to the coupling of modeling and simulation and C4ISR systems, they are necessary in preparing the coupling/integration of different C4ISR systems as well, e.g., to prepare a common operation with new partners and allies. Therefore, it is a general approach to interoperability. In his paper, "Common Data Administration, Data Management, and Data Alignment as a Necessary Requirement for Coupling C4ISR Systems and M\&S Systems," Andreas Tolk outlines some of the work done in this field on international level and draws some conclusions for future work.

The need for interoperability continues to be identified as a crucial element in providing more efficient and effective, multi-national and multi-agency operations. The ability to exchange information, coordinate resources, and understand each participant's capability is paramount to meeting today's challenges on the military and civil battlefields. Joint, combined, multinational training is seen as one key to the transformation required to effect interoperability. According to Ronald J. Roland, a common simulation-training platform, tested and exercised on a regular basis will lead toward interoperability. There are a large number of platform combinations that may prove effective. A potential architecture to consider, presented in his paper "A Small Step toward Interoperability" as an example, is the USAF-ESC National Military Command Center (NMCC) concept combined with the JTLS model, a 
worldwide standard for theater level simulations. The paper addresses the critical issues that have been resolved toward meeting the NMCC requirements of providing a common simulation software environment for both crisis management coordination at the intra and international levels and a potential candidate that can be used for combined, joint and coalition training of combat and security forces. Ronald Roland have provided information and guidelines concerning future enhancements programmed for JTLS and how each user can help guide continued upgrades and revisions.

\section{M\&S Applications}

To illustrate the diversity of possible modeling and simulation applications, even confined only to defense and security, is not an easy task. The papers collected in this group provide just a glimpse at possible application scenarios. With this selection of papers we also try to provide a small but representative selection of possible modeling and simulation technologies and methodologies: fuzzy sets theory, game theory and variational calculus.

At present, the control loops of moving platforms are designed on the base of fuzzy control theory. Especially path searching in a 2D changing environment has received considerable attention as a part of the general problem of robot motion planning. A particularly interesting problem in this context is path planning with respect to a moving object. The design of such intelligent guided vehicles needs capabilities for environment recognition and motion planning. Nowadays, fuzzy control is a promising technique for intelligent system design. The most important feature of this method is that it eliminates the difference between goals and constraints and makes it possible to relate them in the decision-making process. George Georgiev and Valentine Penev propose a fuzzy control method for autonomous guided vehicle, which tracks an object in 3D space. Computer simulations verify the validity and effectiveness of the proposed fuzzy control method. In addition, techniques for path planning in an expanded fuzzy environment, including both stationary and moving obstacles, are under study by the authors.

The second paper, "Game Theoretical Modeling for Planning and Decision-Making," turns the attention to game theory. The author, Juliana Karakaneva, applies this powerful means to modeling of real conflict situations. The approach is well known and established and there are many researchers working in this field. Recently, the importance of these techniques has increased in order to address the necessity to plan and make timely decisions in conditions of incomplete information and in asymmetric environments. In many cases it is impossible to apply mathematical methods due to the difficulties in finding adequate solutions. Modern software for optimization modeling enables to obtain credible models and solutions. 
And finally, the third paper in this group, "Modeling in Shaped Charge Design," demonstrates the use of variational calculus as a tool in the development process of shaped charge geometry. Variational calculus is used for optimization of shaped charges for high-velocities forming of compact, discrete or dispersed jets. Hristo Hristov considers each characteristic function of the shaped charge geometry as a variational parameter in the Orlenko hydrodynamic model. Respectively, the author formulates the problem for determination of an unconditional extremum, as well as a subproblem for determination of a conditional extremum when an integrated condition is added.

\section{M\&S in Information Assurance}

A challenge that stands before the information security community is to better prepare management, system administrators, and users to respond appropriately to information security crises while simultaneously reducing the anxiety associated with them. One clear approach to achieving this goal is to use modeling and simulation for education, training, and testing. The use of $M \& S$ can provide a better understanding of the information environment on both a concrete and abstract level. Proactively it can be used to identify weaknesses and reactively it can provide education and training using "what if" scenarios. Ultimately when new threats are introduced the ability of the organization to respond is significantly enhanced.

This group of papers will try to demonstrate just a small part of the available range of modeling and simulation capabilities in information assurance. It will also attempt to establish some principles for extending these capabilities into the community, and thus to provide a framework for future computer based modeling and simulation efforts in information security.

The first paper in this group treats the issues related to proxy signature. A proxy signature allows a designated person, called a proxy signer, to sign a message on behalf of an original signer. Many proxy signature-related schemes have been proposed due to the importance of this type of scheme. However, as Wei-Bin Lee and Tzung-Her Chen claim, these new schemes always face security challenges. To minimize security challenges, the objective of their research described in the paper "Constructing a Proxy Signature Scheme Based on Existing Security Mechanisms" is to construct a proxy signature scheme that combines existing security mechanisms, rather than attempting to invent a new scheme. Lee and Chen believe that the proposed proxy signature scheme not only satisfies the essential properties mentioned in the well known Mambo-Usuda-Okamoto's proxy signature scheme but also has additional advantages, such as provision of non-repudiation and prevention of delegation transfer. Furthermore, the authors assure that their proxy signature scheme 
does not affect the current security infrastructure and, thus, is more practical than the previously proposed schemes.

Intrusion detection is in the focus of the next paper. However, the authors Andrea Sanna and Claudio Fornaro put the stress more on topics such as mobile devices and visualization. Mobile devices allow a sort of ubiquitous access. This can be of great value to all disciplines, especially in defense and security, where information has to be conveyed to the user in real time independently of his/her physical location. Intrusion detection applications can take advantage of the use of mobile devices by allowing a constant monitoring of the state of a computer system.

Intrusion detection applications often produce large amount of data. The visualization of this information is a key task in order to allow the user to effectively detect attacks and intrusions. Information visualization is an important sub-discipline within the field of scientific visualization and focuses on visual mechanisms designed to communicate clearly to the user the structure of information and to facilitate the access to large data repositories. A new challenge in information visualization is the use of Personal Digital Assistant (PDA) devices.

In their contribution to this special issue, "IMoViS: A System for Mobile Visualization of Intrusion Detection Data," Andrea Sanna and Claudio Fornaro propose an integrated framework to visualize intrusion detection data on PDAs. The proposed architecture is used by a security manager to remotely monitor large buildings for computer intrusion attempts using only a PDA. The Snort ID system is used to detect attacks and intrusions and to store the collected information into a database. The information is processed by software called Guardian that produces the actual data to be fed to the visualization application. From the visualization point of view, this paper presents a graphical interface designed for PDAs. Data related to the building are organized hierarchically; this allows the user to discover and manage intrusions at the top level of the hierarchy, as well as at the leaf level, where detailed information about the attack can be obtained.

Finally, this special issue provides a comprehensive, up-to-date list with on-line resources on general $M \& S$ research and journals; security and defense oriented M\&S research, projects, and software tools, as well as some publications. The I\&S Monitor section contains a description of a simulation on defense resource management held in December 2003 at the Defense and Staff College, Sofia, Bulgaria. The Bulgarian Ministry of Defense organized the event in collaboration with the Institute for Defense Analyses. I\&S Monitor contains also a description of the last report of the NATO RTO Modelling and Simulation Group "C3I and Modelling and Simulation (M\&S) Interoperability." 